\title{
Tumor budding as a risk factor of lymph node metastasis in submucosal invasive T1 colorectal carcinoma: a retrospective study
}

\author{
Bong-Hyeon Kye ${ }^{1}$, Ji-Han Jung ${ }^{2}$, Hyung-Jin Kim', Se-Goo Kang ${ }^{1}$, Hyeon-Min Cho ${ }^{1 *}$ and Jun-Gi Kim ${ }^{3}$
}

\begin{abstract}
Background: This study was designed to identify risk factors for lymph node metastasis of early stage colorectal cancer, which was confirmed to a carcinoma that invaded the submucosa after radical resection.

Methods: In total, 55 patients revealing submucosal invasive colorectal carcinoma on pathology who underwent curative radical resection at the Department of Surgery, St. Vincent's Hospital, The Catholic University of Korea from January 2007 to September 2010 were evaluated retrospectively. Tumor size, depth of submucosal invasion, histologic grade, lymphovascular invasion, tumor budding, and microacinar structure were reviewed by a single pathologist. Student t-test for continuous variables and Chi-square test for categorical variables were used for comparing the clinicopathological features between two groups (whether lymph node involvement existed or not). Continuous variables are expressed as the mean \pm standard error while statistical significance is accepted at $\mathrm{P}<0.05$.

Results: The mean age of 55 patients (34 males and 21 females) was $61.2 \pm 9.6$ years (range, 43-83). Histologically, eight (14.5\%) patients had metastatic lymph node. In the univariate analysis, tumor budding $(P=0.047)$ was the only factor that was significantly associated with lymph node metastasis. Also, the tumor budding had a sensitivity of $83.3 \%$, a specificity of $60.5 \%$, and a negative predictive value of 0.958 for lymph node metastasis in submucosal invasive T1 colorectal cancer.

Conclusions: The tumor budding seems to have a high sensitivity (83.3\%), acceptable specificity (60.5\%), and a high negative predictive value (0.958). A close examination of pathologic finding including tumor budding should be performed in order to manage early CRC properly.
\end{abstract}

Keywords: Lymph node metastasis, T1 colorectal cancer, Tumor budding

\section{Background}

An increase in colorectal cancer (CRC) screening and progress in techniques has resulted greater frequency of detection of T1 stage CRC. With recent advances in endoscopic techniques and improved endoscopes, T1 stage CRC and adenomatous polyps are often resected by endoscopes, regardless of their size or location [1-3]. Local treatments such as endoscopic resection and local excision are considered adequate management for early stage CRC without lymph node metastasis. A complete local treatment of intramucosal carcinoma is accepted as

\footnotetext{
*Correspondence: hmcho@catholic.ac.kr

'Department of Surgery, St. Vincent's Hospital, College of Medicine, The Catholic University of Korea, 93-6, Ji-dong, Paldal-gu, Suwon-si, Gyeonggi-do 442-723, South Korea

Full list of author information is available at the end of the article
}

a curative treatment because there is little risk of lymph node metastasis. However according to the definitions used by most authors, colorectal adenomas with early invasive carcinoma contain a carcinoma that invades the submucosa, but not the muscularis propria. There is a risk of lymph node metastasis when cancer cells have invaded the submucosa. [3-6]. Therefore to avoid overtreatment or under-treatment, histopathological evaluation of locally excised specimens is important.

The previous reported incidence of lymph node metastasis in early stage CRC ranges from 0 to $15.4 \%$, and the risk factors after endoscopic polypectomy include carcinoma at the surgical margin, lymphovascular invasion, poorly differentiated adenocarcinoma, and level of invasion $[7,8]$. Recently, some investigators suggested that a

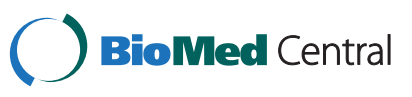


tumor budding is an another risk factor for lymph node metastasis of early stage CRC including occult metastasis $[9,10]$.

This study was designed to identify risk factors for lymph node metastasis of early stage CRC, which was confirmed to a carcinoma that invade the submucosa after radical resection.

\section{Methods}

A total of 55 patients revealing submucosal invasive colorectal carcinoma on pathology who underwent curative radical resection at the Department of Surgery, St. Vincent's Hospital, The Catholic University of Korea from January 2007 to September 2010 were evaluated retrospectively. After obtaining the review board approval from our institute(VC11RISE0171), demographics and pathologic findings of 55 patients were reviewed retrospectively.

In this study, all tumors were macroscopically polypoid type which protruded above the surrounding surface at endoscopic finding. These tumors were classified into three different kinds: Ip, Isp, and Is. Ip is an abbreviation for pedunculated polyp which the base is narrow. Isp is an intermediate and broad-based forms. Lastly, Is is a sessile polyp which the base and the top of the lesion have the same diameter [11]. We defined the right sided colon as cecum, ascending, hepatic flexure, and proximal transverse colon. And the left sided colon was defined as mid to distal transverse, descending, sigmoid, and rectosigmoid colon. The circumferential ratio of the tumor represents the ratio of circumference of tumor to luminal circumference. All pathologic slides were reexamined in which tumor size, depth of submucosal invasion, histologic grade, lymphovascular invasion, tumor budding, and microacinar structure by a single pathologist. The depth of submucosal invasion was defined as the depth between muscularis mucosae and deep tumor margin measured in micrometers. Also, Kudo's classification was used with the relative invasion depth of the submucosal layer and they are as follow: sm1, infiltration into the upper third of the submucosal layer; sm2, middle third; or sm3, lower third [4]. Histologic grade was classified in the order of well, moderately, poorly differentiated, and mucinous adenocarcinoma. Lymphovascular invasion is defined as the presence of tumor cells within small luminal structures lined by endothelial cells. An isolated cell or a small cluster of carcinoma cells in the invasive front is determined by looking at a budding focus, which when viewed at a 200 -fold magnification, > 10 budding foci was considered as positive for tumor budding (Figure 1). Finally microacinar structure specifies small tubules that form cribriform structures within the medium or large glands, or small isolated round tubules within the stroma (Figure 2) [12].

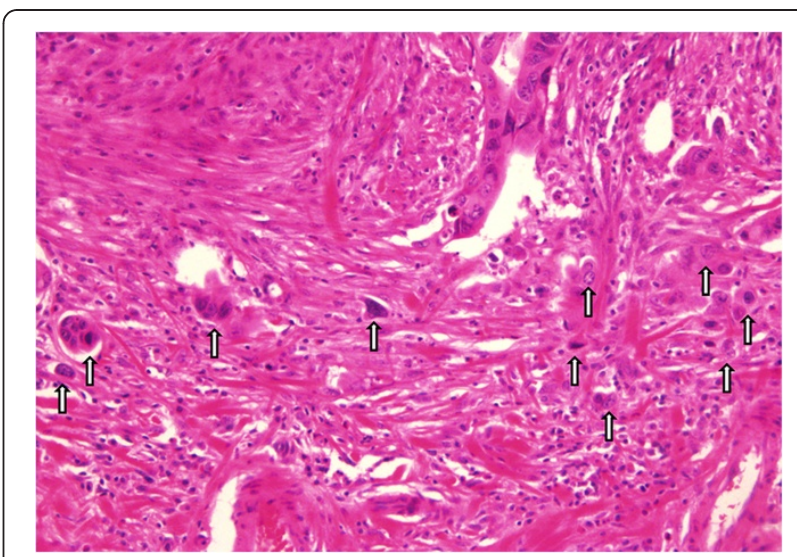

Figure 1 Representative hematoxyline-eosin (H \& E) staining of tumor budding(arrow) - present case (x 200).

Student $t$-test for continuous variables and Chi-square test for categorical variables were used for comparing the clinicopathological features between two groups whether lymph node involvement existed or not. Continuous variables are expressed as the mean \pm standard error whereas statistical significance is accepted at $\mathrm{P}<0.05$. The applied statistical software was $\mathrm{SPSS}^{\circledR} 12.0$ (SPSS Inc., Chicago, IL, USA).

\section{Results}

There were a total of 55 patients including 34 males and 21 females. The mean age of the patients was $61.2 \pm 9.6$ years (range, 43-83). Histologically, eight patients $(14.5 \%)$ had metastatic lymph node. The mean tumor size (the largest diameter) was $2.3 \pm 1.4 \mathrm{~cm}$ (range, 0.7-8.0 cm). Table 1 shows the clinicopathological features in patients with early CRC with submucosal invasion. In the univariate analysis, the lymph node metastasis was significantly associated with the tumor budding $(P=0.047)$ while other factors were not statistically

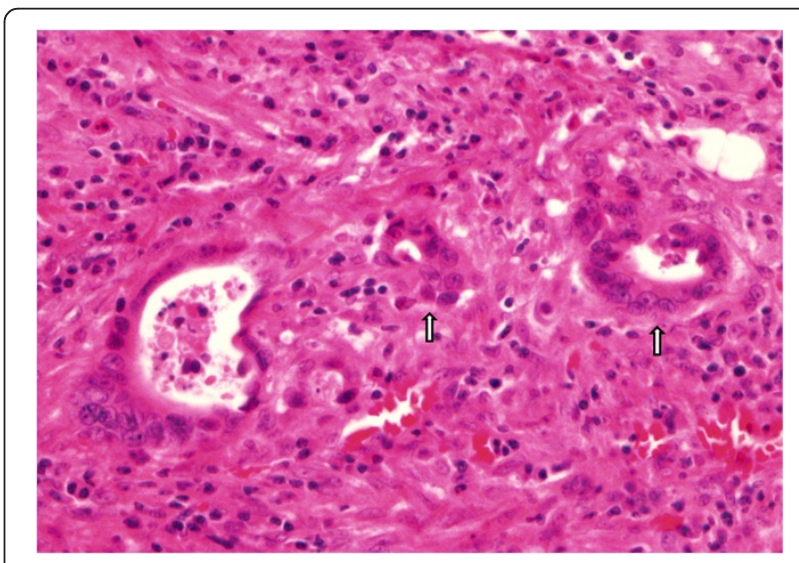

Figure 2 The tumor cells show microacinar structures (small round tubules) (arrow) (H \& E x 400). 
Table 1 Risk factor for lymph node metastasis in patients with submucosal invasive colorectal cancer

\begin{tabular}{|c|c|c|c|c|c|}
\hline & & Lymph & rement & & \\
\hline & & No $(n=47)$ & Yes $(n=8)$ & Univariate & Multivariate \\
\hline Age & Mean \pm S.D. & $60.8 \pm 9.7$ & $64.0 \pm 8.9$ & 0.381 & \\
\hline Sex & male & 29 (61.7\%) & $5(62.5 \%)$ & & \\
\hline & female & 18 (38.3 \%) & $3(37.5 \%)$ & 0.966 & \\
\hline Tumor location & Rt. Sided colon & $6(12.8 \%)$ & 2 (25.0 \%) & & \\
\hline & Lt. Sided colon ${ }^{\S}$ & 22 (46.8 \%) & $4(50.0 \%)$ & & \\
\hline & Rectum & 19 (40.4 \%) & $2(25.0 \%)$ & 0.345 & \\
\hline Macroscopic shape of & Ip & 23 (48.9 \%) & $3(37.5 \%)$ & & \\
\hline tumor & Isp & $9(19.1 \%)$ & $1(12.5 \%)$ & & \\
\hline & Is & $12(25.5 \%)$ & $3(37.5 \%)$ & 0.485 & \\
\hline Tumor size & $\leq 2.3 \mathrm{~cm}$ & 20 (42.6\%) & $5(62.5 \%)$ & & \\
\hline & $>2.3 \mathrm{~cm}$ & 19 (40.4\%) & $2(25.0 \%)$ & 0.428 & \\
\hline Circumference ratio $^{\dagger}$ & $\leq 1 / 4$ & $26(55.3 \%)$ & $6(75.0 \%)$ & & \\
\hline & $1 / 4<$ or $\leq 2 / 4$ & 17 (36.2 \%) & $2(25.0 \%)$ & & \\
\hline & $2 / 4<$ or $\leq 3 / 4$ & $1(2.1 \%)$ & 0 & & \\
\hline & $>3 / 4$ & 0 & 0 & 0.375 & \\
\hline Depth of invasion $(\mu \mathrm{m})$ & $<1000$ & $1(2.1 \%)$ & 0 & & \\
\hline & $1000 \leq$ or $<2000$ & $6(12.8 \%)$ & $2(25.0 \%)$ & & \\
\hline & $2000 \leq$ or $<3000$ & $10(21.3 \%)$ & 0 & & \\
\hline & $3000 \leq$ & 23 (48.9 \%) & $4(50.0 \%)$ & 0.435 & 0.282 \\
\hline Depth of invasion (by & Sm1 & $12(25.5 \%)$ & $2(25.0 \%)$ & & \\
\hline Kudo's classification) & Sm2 & 17 (36.2 \%) & $1(12.5 \%)$ & & \\
\hline & Sm3 & $10(21.3 \%)$ & $3(37.5 \%)$ & 0.364 & 0.063 \\
\hline Differentiation & well & $21(44.7 \%)$ & $1(12.5 \%)$ & & \\
\hline & moderately & 21 (44.7\%) & $6(75.0 \%)$ & & \\
\hline & poorly & 0 & 0 & 0.112 & 0.856 \\
\hline Lymphovascular & Absent & 37 (78.7 \%) & $4(50.0 \%)$ & & \\
\hline invasion & Present & $5(10.6 \%)$ & $2(25.5 \%)$ & 0.206 & 0.232 \\
\hline Tumor budding & Absent & 23 (48.9 \%) & $1(12.5 \%)$ & & \\
\hline & Present & 15 (31.9\%) & $5(75.0 \%)$ & 0.047 & 0.042 \\
\hline Microacinar structure & Absent & $29(61.7 \%)$ & $3(37.5 \%)$ & & \\
\hline & Present & 9 (19.1\%) & $3(37.5 \%)$ & 0.179 & 0.247 \\
\hline
\end{tabular}

$\dagger=$ including cecum, ascending, hepatic flexure, and proximal transverse colon. $\$=$ including mid to distal transverse, descending, sigmoid, and rectosigmoid colon. $\S=$ ratio of the circumference of tumor to luminal circumference.

significant. Also, in the multivariate analysis, the tumor budding $(\mathrm{P}=0.042$, Hazard ratio 13.285, Confidence interval 1.094 - 161.297) was the only independent factor for lymph node metastasis in early CRC with submucosal invasion. The tumor budding had a sensitivity of $83.3 \%$ and a specificity of $60.5 \%$. Also, although the tumor budding had a low positive predictive value (0.25), it had a negative predictive value of 0.958 for lymph node metastasis in submucosal invasive T1 colorectal cancer. Although there was not any statistically significant factor shown in the multivariate analysis, the depth of invasion by Kudo's classification $(P=0.063)$ was a marginally meaningful predictive factor for lymph node metastasis in early CRC with submucosal invasion.

In the present study, 22 of 55 patients underwent colonoscopic polypectomy before curative radical surgery. Table 2 shows the causes of radical surgery after colonoscopic polypectomy. An incomplete polypectomy (45.5\%) was the most frequent reason for a curative radical surgery.

Table 3 shows the clinicopathological features of eight patients who had submucosal invasive CRC with lymph 
Table 2 Causes of radical resection after colonoscopic polypectomy

\begin{tabular}{lc}
\hline \multicolumn{1}{c}{ Causes } & N (=22) \\
\hline Involved or closed resection margin & $10(45.5 \%)$ \\
Patient wanted (Sm1 or Sm2) & $4(18.2 \%)$ \\
No identification of tumor depth, & $3(13.7 \%)$ \\
lymphovascular invasion, or resection margin & \\
Lymphatic invasion (+) & $2(9.1 \%)$ \\
No lifting of tumor at trial for polypectomy & $1(4.5 \%)$ \\
Piecemeal resection & $1(4.5 \%)$ \\
Sessile polyp c rectal invasive carcinoma & $1(4.5 \%)$ \\
\hline
\end{tabular}

node metastasis. Data of malignant polyps in two patients who underwent colonoscopic polypectomy at a local clinic before radical surgery could not be found. The tumor budding was found in five $(83.3 \%)$ of six patients whose pathologic data of malignant polyps could be found. The microacinar structure was found in three $(50 \%)$ of six patients. There was one patient having a recurrence after curative radical surgery. She underwent laparoscopic low anterior resection for malignant rectal polyp located $7 \mathrm{~cm}$ from the anal verge. After the curative surgery, she was given an adjuvant chemotherapy using 5-FU and leucovorin (LF) regimen after curative surgery, but multiple unresectable liver metastases were found 3 months later. Although we gave FOLFOX chemotherapy, the liver metastases progressed and the patient passed away eleven months after radical surgery.

\section{Discussion}

The progress of endoscopic diagnosis and techniques has resulted higher frequency of detection of early stage CRC. With the advances of endoscopic instruments and techniques, the number of endoscopic resections for early-stage CRC has increased providing better quality of life for patients. In the treatment of early CRC which tumor cell forming in mucosal layer, it is better to perform a local treatment without adjuvant therapy because such cancer rarely metastasizes to the lymph node or distant organs [6]. With application of endoscopic treatments being widespread, an adverse outcome such as tumor recurrence was observed occasionally[13,14]. Therefore, this treatment is only adequate for early stage CRC without lymph node metastasis when the cancer cells have invaded submucosa [5-7]. In previous studies reported, the depth of submucosal invasion (Sm3), poor histologic grade, lymphovascular invasion, and tumor cell dissociation are labeled to be the risk factors for lymph node metastasis in submucosal invasive CRC.

Among these factors, the depth of submucosal invasion is an important predictor of lymph node metastasis [4-7,10,13-16]. There are two ways to measure the depth of submucosal invasion. First is to directly measure the vertical distance of submucosal invasion from muscularis mucosae. When the muscularis mucosae could not be identified due to carcinomatous invasion, the superficial aspect of the submucosal invasive carcinoma is used as a baseline. The other is by measuring a relative depth applying the Kudo classification [4]. Kitajima et al. [15] examined 865 submucosal invasive CRCs and analyzed the correlation between lymph node metastasis and depth of submucosal invasion. For pedunculated submucosal invasive CRC, the rate of lymph node metastasis was $0 \%$ in head invasion cases and stalk invasion cases with depth of invasion $<3000 \mu \mathrm{m}$ if lymphatic invasion was negative. For nonpedunculated submucosal invasive CRC, the rate of lymph node metastasis was also $0 \%$ if the depth of invasion was $<1000 \mu \mathrm{m}$. Sung et al. [6] demonstrated that the minimal extent of submucosal invasion in tumors with lymph node metastasis was $1840 \mu \mathrm{m}$. Based on this finding, they suggested that when submucosal invasion of an endoscopically resected

Table 3 Clinicopathological features of eight patients who had T1 colorectal cancer with lymph node metastases

\begin{tabular}{|c|c|c|c|c|c|c|c|c|c|c|c|c|c|c|}
\hline \multirow[t]{2}{*}{ Sex } & \multirow[t]{2}{*}{ Age } & \multirow[t]{2}{*}{ Location } & \multirow[t]{2}{*}{ Shape } & \multirow{2}{*}{$\begin{array}{l}\text { No of } \\
(+) \text { LN }^{+}\end{array}$} & \multirow{2}{*}{$\begin{array}{l}\text { Size } \\
(\mathrm{cm})\end{array}$} & \multirow[t]{2}{*}{ Grade } & \multicolumn{2}{|c|}{ Depth } & \multirow[t]{2}{*}{$\mathrm{LVI}^{\ddagger}$} & \multirow[t]{2}{*}{ Budding } & \multirow[t]{2}{*}{$\mathrm{MA}^{\S}$} & \multirow{2}{*}{$\begin{array}{l}\text { Recurrence } \\
\text { site }\end{array}$} & \multirow{2}{*}{$\begin{array}{c}\text { DFS" } \\
\text { (months) }\end{array}$} & \multirow{2}{*}{$\begin{array}{c}\text { OS } \\
\text { (months) }\end{array}$} \\
\hline & & & & & & & $\mu \mathrm{m}$ & Kudo & & & & & & \\
\hline$F$ & 60 & $\mathrm{~S}^{+\dagger}$ & Isp & 1 & 1.8 & $\mathrm{MD}^{++\dagger}$ & 5000 & Sm3 & - & + & + & No & 54 & 54 \\
\hline$M$ & 64 & $\mathrm{Ra}^{\mathrm{t}}$ & Is & 1 & 1.5 & $\mathrm{MD}^{++\dagger}$ & 1000 & Sm1 & - & + & + & No & 49 & 49 \\
\hline$M$ & 63 & $\mathrm{~S}^{++}$ & Is & 1 & 1.5 & $\mathrm{MD}^{\dagger+\dagger}$ & 3500 & sm3 & - & + & + & No & 37 & 37 \\
\hline$F$ & 62 & $\mathrm{Rb}^{\S \S}$ & Is & 6 & 3.2 & $\mathrm{MD}^{\dagger+\dagger}$ & 1500 & Sm1 & + & + & - & liver & 3 & 11 \\
\hline$M$ & 75 & $A^{\|\| \|}$ & Is & 2 & 1.8 & $\mathrm{MD}^{++\dagger}$ & 3000 & sm3 & - & - & - & No & 15 & 15 \\
\hline$F$ & 78 & $A^{\|\| \|}$ & Ip & 4 & 6 & WD & 3000 & $\mathrm{Sm} 2$ & + & + & - & No & 13 & 13 \\
\hline$M$ & 50 & $R S^{\boldsymbol{\oplus} \oplus}$ & Ip & 2 & 3 & $\mathrm{MD}^{++\dagger}$ & $N R^{\S \S \S}$ & $N R^{\S \S \S}$ & $N R^{\S \S \S}$ & $N R^{\S \S \S}$ & $N R^{\S \S \S}$ & No & 13 & 13 \\
\hline$M$ & 60 & $\mathrm{~S}^{++}$ & Ip & 1 & 1.6 & $\mathrm{MD}^{++\dagger}$ & $N R^{\S \S \S}$ & $N R^{\S \S \S}$ & $N R^{\S \S \S}$ & $N R^{\S \S \S}$ & $N R^{\S \S \S}$ & No & 12 & 12 \\
\hline
\end{tabular}

$\dagger=$ Lymph node, $\$=$ Lymphovascular invasion, $\S=$ Microacinar structure, $\|=$ Disease free survival,

$\uparrow=$ Overall survival, $\dagger+=$ Sigmoid colon, $+\$=$ Rectum above peritoneal reflexion,

$\S \S=$ Rectum below peritoneal reflexion, \|\|$=$ Ascending colon, $\boldsymbol{\top} \Phi=$ Rectosigmoid colon,

$+\dagger+=$ Moderately differentiated, $+t+=$ Well differentiated, $\$ \S \S=$ Not reported. 
tumor was $<1000-1500 \mu \mathrm{m}$, a complete cure can be achieved by endoscopic resection alone. Kudo et al. [4] proposed criteria for staging early CRCs in 1984 classifying degrees of submucosal invasion into three types based on the depth of invasion: sm1 (less than one-third of the submucosa is invaded), sm2 (intermediate), and sm3 (more than two-thirds of submucosa is invaded). For sm1, it is subclassified into sm1 into a, b, and c, according to the degree of horizontal extension of cancer in the submucosal layer. This staging of submucosal invasion is reflected to the prognosis of lesion. They reported that the lymph node metastasis was detected $3.7 \%$ in sm1c cancers, $10.9 \%$ in $\mathrm{sm} 2$ cancers, and $23.7 \%$ in sm3 cancers. In present study, eight patients had metastatic lymph node. However, data of malignant polyps in two patients who underwent colonoscopic polypectomy at a local clinic before radical surgery could not be found. There was no lymph node metastasis in submucosal invasive CRCs that the depth of invasion was $<1000 \mu \mathrm{m}$. In terms of primary tumor with lymph node metastasis, four $(67.7 \%)$ of six patients had a depth of invasion of $\geq 3000 \mu \mathrm{m}$ while two patients had depth of $1000 \mu \mathrm{m}$ and $1500 \mu \mathrm{m}$ respectively. The lymph node metastasis was detected in $14.3 \%$ of sm 1 cancers, $5.6 \%$ of $\operatorname{sm} 2$ cancers, and $23.1 \%$ of $\mathrm{sm} 3$ cancers. Comparing to other previous studies, the lymph node metastasis was more frequently detected in sm1 cancers. Based on this finding, although the depth of invasion is very important predictive factor for lymph node metastasis in submucosal invasive CRC, other factors such as microscopic grade, lymphovascular invasion, and tumor budding should be considered for complete cure by endoscopic resection alone. Ueno et al. [16] demonstrated that to extend the criteria for curative endoscopic resection, the combination of a quantitative factor and qualitative factors is useful. The quantitative factor is the depth of submucoal invasion and the qualitative factors include tumor grade, lymphovascular invasion, tumor budding, etc. Several studies have reported that the lymphovascular invasion is one of the histological risk factors for lymph node metastasis of submucosal invasive CRC $[10,15-17]$. In light of the clinical and biological importance of this feature in T1 tumors, American Joint Committee on Cancer recommended that the following modification of the T1 category (tumor invades the submucosa) to be used: T1a (no evidence of lymphatic or venous invasion) or $\mathrm{T} 1 \mathrm{~b}$ (lymphatic or venous invasion is present). However, it will remain problematic as long as diagnosis continues to differ occasionally among pathologists. Controversies over the detection of lymphovascular invasion arise mainly from the difficulty in visualizing the lymphatic vessel wall. It is difficult to detect lymphovascular invasion with conventional hematoxylin and eosin (HE) staining [18]. To overcome this limitation, Ishii et al.[19] recommended the use of immunostaining with some materials such as monoclonal antibody D2-40 demonstrating that such method would provide important information about the risk of lymph node metastasis and may prove useful in evaluating the necessity of an additional resection after local excision in T1 CRCs. In the present study, lymphovacular invasion was diagnosed in $7(14.6 \%)$ of 48 cases that were examined only with HE staining. Lymph node metastasis could be found in 2 (28.6\%) of these 7 cases. From our results, we conclude that a close evaluation for lymphovascular invasion with immunostaining may be helpful for identifying the risk factor for lymph node metastasis in submucosal invasive CRC. Tumor budding is also seen to be another important parameter in the evaluation of submucosal invasive CRC. This evaluation for tumor budding has merits in that evaluation is possible by ordinary HE staining [20]. Also, tumor budding may be a factor related to lymphovascular invasion. Morodomi et al. [21] have suggested that by making serial sections of specimen, budding is associated with lymphovascular invasion. Moreover, some reports suggest that the most important factor regarding the presence of budding is lymphovascular invasion.[22,23] High-grade tumor budding has been consistently linked to lymph node metastasis, distant metastasis, local recurrence and correlates with the distance of tumor invasion beyond the outer border of the muscularis propria. Tumor budding is proposed as a useful indicator for isolated tumor cells in lymph nodes in patients with early CRCs and could indicate curative surgery in patients with locally excised T1 tumors.[24] Also, microacinar structures (buds of tumor emanating from larger glands) and undifferentiated cells in stroma may be the antecedent stages of lymphatic invasion.[21] Goldstein et al.[12] suggested the association of extensive budding, microacinar structure and undifferentiated cells along the advancing edge with lymph node metastases. To sum up the findings, it is suggested that one of qualitative factors may not be detected independently, but any of those may be detected at progression of tumor. In other words, the detection of any qualitative factor is important in predicting lymph node metastasis. In this study, tumor budding $(\mathrm{P}=0.042$, Hazard ratio 13.285, Confidence interval $1.094-161.297$ ) was the only independent factor for predicting lymph node metastasis. Also, although there was no lymph node metastasis in 15 patients with tumor budding (positive predictive value was 0.25 ) and not appropriated due to small sample size of this study, tumor budding seems to have a high sensitivity (83.3\%), acceptable specificity (60.5\%), and a high negative predictive value $(0.958)$. We think that a high negative predictive value may be more important than a positive predictive value for clinician in order to decide whether an additional radical resection after local excision 
or colonocopic excision for submucosal invasive T1 colorectal cancer would be required or not. This finding may suggested that a close examination of histopathologic finding would provide important information about the risk of lymph node metastasis and may prove useful in evaluating the necessity of an additional resection after local excision in T1 CRC cases.

Our study does have some limitations. It was a retrospective study using the medical records of small number of patients, and the pathologic review of primary tumor could not be performed in all patients. Hence, our results for depth of invasion and lymphovascular invasion as a risk factor for lymph node metastasis were somewhat different compared with previous reported studies. Therefore, a large scaled prospective trial is necessary to verify the management strategy for submucosal invasive CRC.

\section{Conclusion}

A close examination of pathologic finding including quantitative and qualitative factors should be performed for proper management of submucosal invasive CRC. Especially, the tumor budding seems to have a high sensitivity (83.3\%), acceptable specificity (60.5\%), and a high negative predictive value $(0.958)$ in this study. Therefore, we think that the examination for tumor budding after colonoscopic or local excision for submucosal invasive CRC should be performed routinely. If any risk factor is detected, additional oncologic surgery would be necessary.

\section{Competing interests}

The manuscript is an original work and has not been submitted or is under consideration for publication in another journal. The study complies with current ethical consideration. We also confirm that all the listed authors have participated actively in the study, and have seen and approved the submitted manuscript. The authors do not have any possible conflicts of interest.

\section{Author's contributions}

Study conception and design: Kye and Cho. Acquisition of data: Kye, Jung, HJ Kim, Kang, JG Kim, and Cho. Analysis and interpretation of data: Kye, Jung, HJ Kim, Kang, JG Kim, and Cho. Drafting of manuscript: Kye, HJ Kim, JG Kim, and Cho. Critical revision: Kye, Jung, HJ Kim, JG Kim, Kang, and Cho. Final approval : Jung, Kye, Kim, Kang, JG Kim, and Cho.

\section{Author details}

${ }^{1}$ Department of Surgery, St. Vincent's Hospital, College of Medicine, The Catholic University of Korea, 93-6, Ji-dong, Paldal-gu, Suwon-si, Gyeonggi-do 442-723, South Korea. 'Department of Pathology, St. Vincent's Hospital, College of Medicine, The Catholic University of Korea, 93-6, Ji-dong, Paldal-gu, Suwon-si, Gyeonggi-do 442-723, South Korea. ${ }^{3}$ Department of Surgery, Seoul St. Mary's Hopsital, College of Medicine, The Catholic University of Korea, 505 Banpo-dong, Seocho-gu, Seoul, Korea.

Received: 16 January 2012 Accepted: 3 August 2012

Published: 6 August 2012

\section{References}

1. Kudo S, Tamegai Y, Yamano H, Imai Y, Kogure E, Kashida H: Endoscopic mucosal resection of the colon: the Japanese technique. Gastrointest Endosc Clin N Am 2001, 11:519-535.
2. Yamamoto $H$, Koiwai $H$, Yube $T$, Isoda $N$, Sato $Y$, Sekine $Y$, Higashizawa T, Utsunomity K, Ido K, Sugano K: A successful single-step endoscopic resection of a 40 millimeter flat-elevated tumor in the rectum: endoscopic mucosal resection using sodium hyaluronate. Gastrointest Endosc 1999, 50:701-704.

3. Hurlstone DP, Sanders DS, Cross SS, Adam I, Shorthouse AJ, Brown S, Drew K, Lobo AJ: Colonoscopic resection of lateral spreading tumours: a prospective analysis of endoscopic mucosal resection. Gut 2004, 53:1334-1339.

4. Kudo $S$, Kashida H, Tamura $T$, Kogure $E$, Imai $Y$, Yamano H, Hart AR: Colonoscopic diagnosis and management of nonpolypoid early colorectal cancer. World J Surg 2000, 24:1081-1090.

5. Nivatvongs S: Surgical management of early colorectal cancer. World J Surg 2000, 24:1052-1055.

6. Sung HY, Kang WK, Kim SW, Nam KW, Jung CK, Chang JH, Cho YK, Park JM, Lee IS, Lee JI, Oh ST, Choi MK, Chung IS: Risk factors for lymph node metastasis in patients with submucosal invasive colorectal carcinoma. J Korean Surg Soc 2010, 78:207-212.

7. Kikuchi R, Takano M, Takagi K, Fujimoto N, Nozaki R, Fujiyoshi T, Uchida Y: Management of early invasive colorectal cancer; Risk of recurrence and clinical guidelines. Dis Colon Rectum 1995, 38:1286-1295.

8. Rossini FP, Ferrari A, Coverlizza S, Spandre M, Risio M, Gemme C, Cavallero M: Large bowel adenomas containing carcinoma-a diagnostic and therapeutic approach. Int J Colorectal Dis 1988, 3:47-52.

9. Yasuda K, Inomata M, Shiromizu A, Shiraishi N, Higashi H, Kitano S: Risk factors for occult lymph node metastasis of colorectal cancer invading the submucosa and indications for endoscopic mucosal resection. Dis Colon Rectum 2007, 50:1370-1376.

10. Ishikawa Y, Akishima-Fukasawa Y, Ito K, Akasaka Y, Yokoo T, Ishii T: Toho Study Group for Cancer Biological Behavior: Histopathologic determinants of regional lymph node metastasis in early colorectal cancer. Cancer 2008, 112:924-933.

11. : The Paris endoscopic classification of superficial neoplastic lesions: esophagus, stomach, and colon. Gastrointest Endosc 2003, 58:S3--S43. November 30 to December 1, 2002.

12. Goldstein NS, Hart J: Histologic features associated with lymph node metastasis in stage T1 and superficial T2 rectal adenocarcinomas in abdominoperineal resection specimen: Identifying a subset of patients for whom treatment with adjuvant therapy or completion abdominoperineal resection should be considered after local excision. Am J Clin Pathol 1999, 111:51-58.

13. Volk EE, Goldblum JR, Petras RE, Carev WD, Fazio WW: Management and outcome of patients with invasive carcinoma arising in colorectal polyps. Gastroenterology 1995, 109:1801-1807

14. Seitz U, Bohnacker S, Seewald S, Thonke F, Brand B, Bräutigam T, Soehendra $\mathrm{N}$ : Is endoscopic polypectomy an adequate therapy for malignant colorectal adenomas? Presentation of 114 patients and review of the literature. Dis Colon Rectum 2004, 47:1789-1797.

15. Kitajima K, Fujimori T, Fujii S, Takeda J, Ohkura Y, Kawamata H, Kumamoto T, Ishiguro S, Kato Y, Shimoda T, Iwashita A, Ajioka Y, Watanabe H, Watanabe T, Muto T, Nagasako K: Correlations between lymph node metastasis and depth of submucosal invasion in submucosal invasive colorectal carcinoma: a Japanese collaborative study. J Gastroenterol 2004, 39:534-543.

16. Ueno H, Mochizuki H, Hashiguchi Y, Shimazaki H, Aida S, Hase K, Matsukuma S, Kanai T, Kurihara H, Ozawa K, Yoshimura K, Nekku S: Risk factors for an adverse outcome in early invasive colorectal carcinoma. Gastroenterology 2004, 127:385-394.

17. Nascimbeni R, Burgart LJ, Nivatvongs S, Larson DR: Risk of lymph node metastasis in T1 carcinoma of the colon and rectum. Dis Colon Rectum 2002, 45:200-206.

18. Compton C, Fenoglio-Preiser CM, Pettigrew N, Fielding LP: American joint committee on cancer prognostic factors consensus conference: colorectal working group. Cancer 2000, 88:1739-1757.

19. Ishii M, Ota M, Saito S, Kinugasa Y, Akamoto S, Ito I: Lymphatic vessel invasion detected by monoclonal antibody D2-40 as a predictor of lymph node metastasis in T1 colorectal cancer. Int J Colorectal Dis 2009, 24:1069-1074.

20. Fujimori T, Fujii S, Saito N, Sugihara K: Pathological diagnosis or early colorectal carcinoma and its clinical implications. Digestion 2009, 79:40-51. 
21. Morodomi T, Isomoto H, Shirouzu K, Kakegawa K, Irie K, Morimatsu M: An index for estimating the probability of lymph node metastasis in rectal cancers: lymph node metastasis and the histopathology of actively invasive regions of cancer. Cancer 1989, 63:539-543.

22. Okuyama T, Oya M, Ishikawa H: Budding as a risk factor for lymph node metastasis in PT1 or pT2 well-differentiated colorectal adenocarcinoma. Dis Colon Rectum 2002, 45:628-634.

23. Okuyama T, Oya M, Ishikawa $\mathrm{H}$ : Budding as a useful prognostic marker in pT3 well- or moderately-differentiated rectal adenocarcinoma. J Surg Oncol 2003, 83:42-47.

24. Zolbec I, Lugli A: Epithelial mesenchymal transition and tumor budding in aggressive colorectal cancer: Tumor budding as oncotarget.

Oncotarget 2010, 1:651-661.

\section{doi:10.1186/1471-2482-12-16}

Cite this article as: Kye et al: Tumor budding as a risk factor of lymph node metastasis in submucosal invasive T1 colorectal carcinoma: a retrospective study. BMC Surgery 2012 12:16.

\section{Submit your next manuscript to BioMed Central and take full advantage of:}

- Convenient online submission

- Thorough peer review

- No space constraints or color figure charges

- Immediate publication on acceptance

- Inclusion in PubMed, CAS, Scopus and Google Scholar

- Research which is freely available for redistribution 